\title{
DYNAMICAL EVOLUTION OF GALACTIC CENTER STAR CLUSTER AND IMPORTANCE OF STELLAR COLLISIONS
}

\author{
HYUNG MOK LEE \\ Pusan University \\ Department of Earth Sciences, Pusan 609-735, Korea
}

\section{Introduction}

The Galactic Center (GC) star cluster is a compact stellar system. The surface brightness distribution at infrared has core radius of about $0.8 \mathrm{pc}$ (Rieke \& Lebovsky 1987) when the contribution from bright stars are removed. On the other hand number counts of infrared sources in the high resolution speckle image by Eckart et al. (1993) at $2.2 \mu \mathrm{m}$ gives $0.15 \mathrm{pc}$ for the core radius of the surface density distribution. At present it is not clear which is more appropriate for the mass distribution. If we assume that the central star cluster is an isothermal sphere, the central density and the core radius are related via

$$
\rho_{c}=\frac{9 \sigma^{2}}{4 \pi G r_{c}^{2}} \approx \begin{cases}2.7 \times 10^{6} \mathrm{M}_{\odot} \mathrm{pc}^{-3} & \text { if } r_{c}=0.8 \mathrm{pc} \\ 7.4 \times 10^{7} \mathrm{M}_{\odot} \mathrm{pc}^{-3} . & \text { if } r_{c}=0.15 \mathrm{pc} .\end{cases}
$$

where we have assumed one-dimensional velocity dispersion $\sigma=100 \mathrm{~km} / \mathrm{sec}$. Therefore the central density depends sensitively on the measured value of the core radius. The central relaxation time of the cluster also varies greatly depending on the central density,

$$
t_{r e l} \approx \begin{cases}6 \times 10^{8} \mathrm{yrs}, & \text { if } r_{c}=0.8 \mathrm{pc} \\ 2 \times 10^{7} \mathrm{yrs}, & \text { if } r_{c}=0.15 \mathrm{pc} .\end{cases}
$$

where we have assumed that the mass of individual star is $1 \mathrm{M}_{\odot}$. Since core collapse takes place in many multiple of the central relaxation time (i.e., 20 300, e.g., Cohn 1980) one does not expect much evolution during Hubble time if the core radius is $0.8 \mathrm{pc}$ while significant evolution should have occurred if the core radius is $0.15 \mathrm{pc}$. 
For the case of high stellar density core (i.e., $r_{c}=0.15 \mathrm{pc}$ ), one also expects very frequent stellar collisions. The consequences of close encounters depend on the relative velocity at infinity $\left(v_{r e l}\right)$; merger for $v_{r e l}<v_{e s c}$ and complete disruption for $v_{r e l}>v_{e s c}$, where $v_{e s c}$ is the escape velocity from the stellar surface. Since $\sigma \approx 100 \mathrm{~km} / \mathrm{sec}$ in the GC, merger is a natural consequence of stellar collisions. It should be noted that the relaxation time is usually short if the collision time is short. This means that the dynamical evolution must be taken into account if one wants to consider the time dependent stellar mergers.

Recently Brown \& Bethe (1994) proposed that stars with main-sequence mass greater than $\sim 25 \mathrm{M}_{\odot}$ would leave black holes of $\sim 10 \mathrm{M}_{\odot}$ behind at the end of stellar evolution. Such black holes would comprise around 0.5 $\sim 1 \%$ of the stellar mass if Salpeter mass function with relatively large high mass cut-off is assumed. Even such a small amount of black holes could be dynamically important as discussed by Lee (1995).

In this review, we discuss two aspects of the dynamical evolution of GC star cluster: (a) effects of the stellar merger and (b) effects of $10 \mathrm{M}_{\odot}$ black holes.

\section{Dynamical Evolution with Successive Mergers}

The stellar merger may be regarded as an alternative process of forming high mass stars. About $15 \mathrm{HeI} / \mathrm{HI}$ emission line stars have been identified in GC region (e.g., Krabbe et al. 1991) and they are interpreted as $20 \sim$ $40 \mathrm{M}_{\odot}$ stars by Najarro et al. (1993). Since lifetimes of these stars are fairly short, there might have been star formation activity in the past $10^{7}$ years. However, the strong tidal field in GC region requires very high density $\left(n_{H} \gtrsim 10^{7} \mathrm{~cm}^{-3}\right)$ for gravitational collapse. Such high density clouds are not completely ruled out by the present observations (Jackson et al. 1993), but there is no convincing evidence for the star formation activity either.

Is it possible that the high mass stars are born outside the center and segregated to the present location via dynamical friction? The answer is clearly no because the time scale for dynamical friction is longer than the lifetime of these stars. Thus the difference in the core radii for surface brightness distribution and surface density distribution has not been caused by the equipartition process alone. We examine merger scenario as an alternative for the formation of high mass stars below.

\subsection{MODELS FOR SUCCESSIVE MERGERS}

The formation process of high mass stars via stellar mergers may be modeled by simple rate equations (Lee 1989). However, other dynamical effects 
such as relaxation and dynamical friction should be taken into account. One also has to consider the fact that stars live only finite amount of time.

Rather crude modeling for successive mergers including relaxation, dynamical friction, and evolution of high mass stars was introduced by Lee (1987) in the context of globular cluster evolution by integrating (modified) Fokker-Planck equation. Application of this method to denser stellar systems of galactic nuclei was done by Quinlan \& Shapiro (1990). The main purpose of these studies are to test the possibility of 'runaway merger' which could lead to the formation of a central black hole followed by the formation of very massive star which is dynamically unstable.

More specific study towards the GC in an attempt to estimate the number of high mass stars formed through successive merger was performed by Lee (1994). The result of this study is negative since the number of stars exceeding $20 \mathrm{M}_{\odot}$ is of order of 1 at most. Because of indirect heating effect due to the stellar evolution, the core collapse stops at some point and the cluster begins to expand as the heating rate eventually exceeds the rate of conductive outflow of the energy. Numbers of merger products reach maxima just after the maximum density.

The number of high mass stars is rather sensitive to the stellar lifetime. The stars formed by successive mergers may be rapidly rotating and the stellar lifetime could be longer than non-rotating stars. In Table 1, we have compared the maximum number of stars formed by mergers for two different assumptions in lifetimes: the case with standard values and the case with twice the standard values. Other model parameters are chosen to resemble the GC (see Lee 1994 for details). As can be seen from this table, the number of stars with $>20 \mathrm{M}_{\odot}$ can be as large as 5 . The observed number of HeI/HI stars of about 15 is larger by only a factor of few. Since the $\mathrm{HeI} / \mathrm{HI}$ stars are in temporary phase of the stellar evolution, actual number of $M>20 \mathrm{M}_{\odot}$ stars are likely to be much larger then 15 . Thus the successive merger does not appear to be a dominant process of forming high mass stars.

TABLE 1. Maximum Number of Stars Formed by Mergers

\begin{tabular}{ccc}
\hline Mass $\left(\mathrm{M}_{\odot}\right)$ & Standard Lifetime & $2 \times$ Standard Lifetime \\
\hline 1.4 & $4.5 \times 10^{5}$ & $4.6 \times 10^{5}$ \\
2.8 & $2.0 \times 10^{4}$ & $2.6 \times 10^{4}$ \\
5.6 & 940 & 1960 \\
11.2 & 30 & 160 \\
22.4 & 0.3 & 5.3 \\
\hline
\end{tabular}


In the Fokker-Planck calculations, we have assumed that the mass lost by stellar evolution would leave the entire cluster in a short amount of time. If the gas can stay in the vicinity of cluster's core, the heating rate would not be enough to stop the core collapse. The stellar merger then would take place in an accelerated manner. It is possible to have much more high mass stars $\left(M>20 \mathrm{M}_{\odot}\right)$ than the values tabulated in Table 1 . However, the core radius of the cluster should be very small.

\subsection{SHORTFALLS AND POSSIBLE SOLUTIONS}

There are two serious problems in the merger scenario for the formation of high mass stars. First, in order to form $20 \mathrm{M}_{\odot}$ stars, there must be a much larger number of slightly lower mass stars (i.e., $10 \mathrm{M}_{\odot}$ ). Table 1 shows the maximum number of $11.2 \mathrm{M}_{\odot}$ stars can be 50 to 100 times larger than that of $22.4 \mathrm{M}_{\odot}$ stars. These stars could be observed from the GC region. Second, the velocity dispersion of the high mass stars should be smaller than the 'average' velocity dispersion. Since stellar merger is completely inelastic process, the velocity dispersion of the merged stars dictates that of the equipartition (i.e., $m_{1}<v_{1}^{2}>=m_{2}<v_{2}^{2}>$ ). Such a behavior of velocity dispersion has not been observed.

If there is a black hole in the center of the Galaxy, the velocity dispersion would not depend on the mass. The stellar distribution within $r_{b}=G M_{B} / v^{2}$ would follow $r^{-7 / 4}$ (e.g., Bahcall \& Wolf 1976). The collisional frequency then becomes much larger than that for the case with flat core. Actual calculation of stellar merger with a central black hole has not been carried out yet, but it would be interesting to see if the presence of black hole would solve the problems mentioned above.

\section{Effects of Remnant Stars}

The stellar evolution inevitably produces remnant stars such as white dwarfs, neutron stars and black holes. The white dwarfs may not give important effects on dynamical evolution but neutron stars and black holes could be important.

If there are significant number of $10 \mathrm{M}_{\odot}$ black holes in the GC, dynamical friction will bring the black holes into the central parts in $\left(m_{*} / m_{B}\right) t_{r e l} \ll$ $t_{r e l}$, where $m_{*}$ and $m_{B}$ are masses of normal stars and black holes, respectively. After the formation of a black hole subsystem, it evolves quickly because the relaxation time becomes very short. There are many interesting physical processes within the black hole clusters. 


\subsection{BLACK HOLE BINARIES AND DYNAMICAL EVOLUTION}

After the subsystem of black hole is formed, the black hole cluster undergoes core collapse like ordinary stellar systems. Binaries via three-body processes can form rather efficiently because the rate of binary formation is proportional to $m_{B}^{5} n_{B}^{3} \sigma_{B}^{-9}$, where $n_{B}$ is the number density and $\sigma_{B}$ is the velocity dispersion of the black holes.

The binaries become heat source when they interact with other black holes. The core collapse eventually stops and the central core of black hole subsystem begins to expand. The 'post-collapse' expansion driven by binaries formed via three-body processes (three-body binaries in short) can be characterized by self-similar evolution for the case of single-component, isolated clusters. The core and the half-mass radii expand at the same rate. However, since the black hole cluster is embedded in a much larger stellar cluster, the time scale for the expansion is set by the half-mass relaxation time of the surrounding cluster which is much longer than the Hubble time. Thus the energy released from the black hole binaries can be absorbed by the stellar cluster. It might be possible to maintain nearly static two-component structure for a very long time as discussed by Goodman \& Lee (1989). Since the size of the black hole cluster is very small, the cluster of black holes may be indistinguishable from a single massive black hole in the center.

However, there may be a destabilizing affect. Ordinarily binaries are eventually ejected from the stellar system when the hardness (ratio of orbital kinetic energy to average kinetic energy of background stars) becomes very large ( $\sim$ a few 100). For the tight black hole binaries the gravitational radiation effect can be quite important. In the stellar system with $\sigma \gtrsim 100 \mathrm{~km} / \mathrm{sec}$ the binaries are found to 'merge' due to gravitational radiation reaction before they are ejected.

Lee (1993) showed that a runaway merger would produce a 'seed' black hole in the center of a dense stellar system composed of neutron stars. Such a runaway merger is also a possible product in the black hole subsystem if the the 'merger time scale' is short.

Numerical modeling for two-component clusters composed of $0.7 \mathrm{M}_{\odot}$ main-sequence stars and $10 \mathrm{M}_{\odot}$ black holes was carried out by Lee (1995) using Fokker-Planck equation. Heating by three-body binaries enables the post-collapse evolution, which is characterized by rapid oscillations of core density. The black hole cluster expands rather slowly because the surrounding star cluster absorbs the energy.

It is difficult to judge if the runaway merger is possible because of the statistical nature of Fokker-Planck calculations. More realistic N-body calculations are necessary to determine the merger instability. 
If a seed black hole of mass comparable the core mass of the black hole subsystem forms, it can grow by absorbing surrounding black holes and stars.

\subsection{OBSERVATIONAL CONSEQUENCES}

If the two-component phase of star-black hole configuration is long-lived, black hole subsystem may be kinematically similar to a massive central black hole. One of the argument against the massive black hole in the GC is the lack of X-ray radiation (e.g., Ozernoy 1994). Similar arguments can be applied to the two-component cluster for GC (and possibly for other nearby galaxies). The least violent interaction between a black hole and a mainsequence star will be tidal capture. Large fraction of tidally captured mainsequence stars will be destroyed during the circularization process because the energy to be deposited to the normal star exceeds star's gravitational binding energy if mass ratio between the black hole and the normal star is large (Lee 1992). We now estimate the rate of tidal capture.

Suppose that the black hole cluster is embedded in a flat core of normal stars. Then the total number of tidal capture per unit time can be expressed as

$$
\frac{d N_{t c}}{d t}=\int_{V} n_{*} n_{B}<\Sigma_{t c} v_{r e l}>d V \approx n_{*} N_{B}<\Sigma_{t c} v_{r e l}>,
$$

where $n_{*}$ and $n_{B}$ are number densities of normal stars and black holes, respectively, $N_{B}$ is the total number of black holes, and $\Sigma_{t c}$ is the tidal capture cross section. The relative velocity between a normal star and a black hole can be replaced by the velocity of normal star, and the brackets represent the average taken over the velocity distribution. Using the overlap integrals for tidal interactions tabulated by Lee \& Ostriker (1986), we obtain $R_{\text {min }} \approx 8.6 R_{*}$ for $m_{B} / m_{*} \approx 14$ (i.e., $m_{*} \approx 0.7 \mathrm{M}_{\odot}$ ) and $v_{r e l}=100 \mathrm{~km} / \mathrm{sec}$. If we assume that the cross section is dominated by gravitational focusing we may write

$$
\begin{aligned}
\frac{d N}{d t} & \approx 8.6 \pi R_{*} n_{*} N_{B} 2 G m_{B}\left\langle\frac{100 \mathrm{~km} / \mathrm{sec}}{v_{r e l}}\right\rangle \\
& \approx 5 \times 10^{-6}\left(\frac{n_{*}}{10^{6} \mathrm{pc}^{-3}}\right)\left(\frac{N_{B}}{10^{4}}\right)\left(\frac{100 \mathrm{~km} / \mathrm{sec}}{\sigma}\right) \mathrm{yr}^{-1}
\end{aligned}
$$

where we have assumed $R_{*}=0.7 \mathrm{R}_{\odot}$. If we accumulate the above rate over Hubble time, the number of black hole binary would be over $10^{4}$ for GC. Such a large number of binaries may not be compatible with the observations. 
If the large fraction of tidal capture leads to the disruption of mainsequence stars, more appropriate quantity is the instantaneous luminosity from accretion. Some fraction $(\lesssim 50 \%)$ of the gas released from the star will be bound to the black hole. They will eventually be accreted to the black hole and radiation will be released. The tidal capture rate estimated above can thus be translated into the upper limit of $2.5 \times 10^{7} \mathrm{~L}_{\odot}$ on accretion luminosity. Here we have assumed efficiency of $10 \%$ for the conversion of rest mass energy into radiation. Clearly this is also too large compared to the upper limit of $250 \mathrm{~L}_{\odot}$ on X-ray luminosity see Genzel et al. 1994 for a summary). However, the total UV luminosity of about $10^{7} \mathrm{~L}_{\odot}$ is not much different from the accretion luminosity estimated above. The central cluster of hot stars are assumed to be liable for the entire UV luminosity, but it is tempting to postulate that the HeI/HI stars are Thorn-Zytkow type (Thorne \& Zytkow 1977) accretion flows around the black holes (Morris 1993).

\section{Summary}

The observed characteristic of the GC cluster is examined in view of dynamical evolution theories. If the core radius of the mass distribution is as small as $0.15 \mathrm{pc}$, one expects rather frequent collisions between stars. High mass stars can be formed through successive mergers. However, we found that the number of high mass stars $\left(\gtrsim 20 \mathrm{M}_{\odot}\right)$ are not enough to explain $\mathrm{HeI} / \mathrm{HI}$ stars observed in GC region. Also the merger scenario predicts small velocity dispersion for high mass stars than what is observed. These difficulties may be relieved by the introduction of central black hole, but actual calculation has not been carried out yet.

The possible effects of $10 \mathrm{M}_{\odot}$ black holes, which might have formed in early history of the Galaxy through the collapse of He core of high mass stars, are examined. These black holes would evolve separately by forming a subsystem within the flat core of star cluster. The black hole cluster undergoes rapid core oscillation after the core collapse but the general expansion is very slow because the surrounding star cluster effectively absorbs the expansion energy generated by black hole binaries. During this near static two-component phase, a central seed black hole might form through runaway merger of black holes followed by formation of binaries via three-body processes.

If the runaway merger does not take place within Hubble time, the twocomponent cluster should give some interesting observational consequences. First, the kinematic signature of central massive black hole will also be observable because the size of black hole subsystem is very small. Second, the tidal interactions between main-sequence stars and black holes will 
inevitably lead to the accretion of stellar mass. The estimated upper-limit for accretion luminosity is close to the total UV luminosity in the central cluster of hot luminous stars.

\section{Acknowledgements}

This work was supported in part by the Korea Science Engineering Foundation under Grant No. 941-0200-001-2 and in part by National Science Foundation under Grant No. PHY89-04035.

\section{References}

Bahcall, J. N., \& Wolf, R. A., 1976, ApJ, 209, 214.

Brown, G. E., \& Bethe, H. A., 1994, ApJ, 423, 659.

Cohn, H., 1980, ApJ, 242, 765.

Eckart, A., Genzel, R., Hofmann, R., Sams, B. J., Tacconi-Garman, L. E., 1993a, ApJ, $122,433$.

Genzel, R., Hollenbach, D., \& Townes, C. H., 1994, Rep. Prof. Phys, 57, 417.

Goodman, J., \& Lee, H. M., 1989, ApJ, 337, 84.

Jackson, J. M., et al. 1993, ApJ, 402, 173.

Krabbe, A., Genzel, R., Drapatz, S., \& Rotaciuc, V., 1991, ApJ, 382, L19.

Lee, H. M., 1987, ApJ, 319, 801.

-., 1989, in Dynamics of Dense Stellar Systems, ed. D. Merritt, Cambridge Univ. Press, p105. 1992, J. Korean Ast. Soc., 25, 47.

Lee, H. M., 1994, in Nuclei of Normal Galaxies: Lessons from Galactic Center, eds. by R. Genzel \& A. I. Harris, (Dordrecht:Kluwer) in press.

Lee, H. M., 1995, MNRAS, in press.

Lee, H. M., \& Ostriker, J. P., 1986, ApJ, 322, 123.

Lee, M. H., 1993, ApJ, 418, 147.

Morris, M., 1993, ApJ, 408, 496.

Najarro, F., Hillier, D. J., Kudritzki, R. P., Krabbe, A., Lutz, D., Genzel R., Drapatz, S., \& Geballe, T. R., 1993, A\&Ap,

Ozernoy, 1994, in Nuclei of Normal Galaxies: Lessons from Galactic Center, eds. by R. Genzel \& A. I. Harris, (Dordrecht:Kluwer) in press.

Quinlan, G. D., \& Shapiro, S. L., 1990, ApJ, 356, 483.

Rieke, G. H., \& Lebovsky, M. J., 1987, in The Galactic Center, AIP Conference Series, No. 155, ed. D. C. Backer, p91.

\section{DISCUSSION}

J. Grindlay: Let me point out that your estimate of $\sim 10^{4}$ (to even $\sim 10^{5}$ ) as a possible total number of $\sim 10 \mathrm{M}_{\odot} \mathrm{BH}$ remnants in the central cluster is unlikely - since in this case formation of LMXBs containing a $\sim 10 \mathrm{M}_{\odot}$ $\mathrm{BH}$ and $\lesssim 1 \mathrm{M}_{\odot}$ companion would be expected and these, in turn, would be detected as either (or both) persistent and transient bright $\left(\geq 10^{37}\right.$ ergis) $x$-ray sources. In particular, these would show up as "x-ray novae", whereas in $\sim 20$ year of $\mathrm{x}$-ray coverage (since UHURU) no such transients 
(now recognized to be BH LMXBs) have been detected from the Galactic Center. Similar constraints can be derived for the central cusp of neutron stars, although these are less likely to form and to be retained given their power mass and higher velocity than for $\sim 10 \mathrm{M}_{\odot} \mathrm{BHs}$.

Lee: The expected number of black holes is $\sim 10^{3}$. Still you expect quite frequent encounters between black holes and normal stars. Because of large mass ratio between black holes and normal stars, and because of high velocity environment, the likely consequence of $\mathrm{BH}$-normal star interaction is a total disruption of the star. Accretion of disrupted material is likely, but there are many uncertainties regarding physical processes after the disruption.

R. Sunyaev: Did you compute neutron star distribution? If they are numerous $\sim 10^{5}$ in the central cluster, they must form some amount of binaries with strong accretion. We do not see with GRANAT no traces and no place for such bright close as Neutron Star-normal star or Black Hole-normal star $\mathrm{x}$-ray emitting binaries. At the same time they are observed practically in every globular cluster.

Lee: I haven't included neutron stars here. First of all, since neutron stars are much less massive than blackholes, their distribution will be less concentrated than black holes. Second, the probability of forming binaries is very small. The stars will be just disrupted as a result of close encounters. 